\title{
Dosimetric comparison of axilla and groin radiotherapy techniques for high-risk and locally advanced skin cancer
}

\author{
Malcolm D. Mattes, MD*, Ying Zhou, MS², Sean L. Berry, PhD², Christopher A. Barker, MD \\ Departments of ${ }^{1}$ Radiation Oncology and ${ }^{2}$ Medical Physics, Memorial Sloan Kettering Cancer Center, New York, NY, USA
}

Purpose: Radiation therapy targeting axilla and groin lymph nodes improves regional disease control in locally advanced and high-risk skin cancers. However, trials generally used conventional two-dimensional radiotherapy (2D-RT), contributing towards relatively high rates of side effects from treatment. The goal of this study is to determine if three-dimensional conformal radiation therapy (3D-CRT), intensity-modulated radiation therapy (IMRT), or volumetric-modulated arc therapy (VMAT) may improve radiation delivery to the target while avoiding organs at risk in the clinical context of skin cancer regional nodal irradiation.

Materials and Methods: Twenty patients with locally advanced/high-risk skin cancers underwent computed tomography simulation. The relevant axilla or groin planning target volumes and organs at risk were delineated using standard definitions. Paired t-tests were used to compare the mean values of several dose-volumetric parameters for each of the 4 techniques.

Results: In the axilla, the largest improvement for 3D-CRT compared to 2D-RT was for homogeneity index (13.9 vs. 54.3), at the expense of higher lung $\mathrm{V}_{20}(28.0 \%$ vs. $12.6 \%)$. In the groin, the largest improvements for 3D-CRT compared to 2D-RT were for anorectum $D_{\text {max }}$ (13.6 vs. 38.9 Gy), bowel $D_{200 c c}\left(7.3\right.$ vs. 23.1 Gy), femur $D_{50}\left(34.6\right.$ vs. 57.2 Gy), and genitalia $D_{\max }(37.6$ vs. 51.1 Gy). IMRT had further improvements compared to 3D-CRT for humerus $D_{\text {mean }}\left(16.9\right.$ vs. 22.4 Gy), brachial plexus $D_{5}(57.4$ vs. 61.3 Gy), bladder $D_{5}(26.8$ vs. $36.5 \mathrm{~Gy})$, and femur $\mathrm{D}_{50}$ (18.7 vs. $\left.34.6 \mathrm{~Gy}\right)$. Fewer differences were observed between IMRT and VMAT.

Conclusion: Compared to 2D-RT and 3D-CRT, IMRT and VMAT had dosimetric advantages in the treatment of nodal regions of skin cancer patients.

Keywords: Intensity-modulated radiotherapy, Skin neoplasm, Conformal radiotherapy, Merkel cell carcinoma, Melanoma, Squamous cell carcinoma

\section{Introduction}

In locally advanced and high-risk skin cancer, radiotherapy targeting the regional lymph node basin is an effective treatment as demonstrated in randomized trials in cutaneous melanoma and Merkel cell carcinoma [1,2]. In high-risk, stage III cutaneous melanoma, adjuvant node basin radiotherapy to a dose of $48 \mathrm{~Gy}$ in 20 fractions (after therapeutic lymphadenectomy) significantly reduced the cumulative incidence of lymph node relapse from 31\% to $19 \%$ at 3 years, according to the Trans-Tasman Radiation Oncology Group (TROG) [1]. In stage I Merkel cell carcinoma, elective node basin radiotherapy to a dose of $50 \mathrm{~Gy}$ in 25 fractions significantly reduced the likelihood of regional recurrence from $16.7 \%$ to $0 \%$ at 3 years, according to the Groupe de Cancérologie Cutanée of the Sociéte Française de Dermatologie (GCCSFD) [2]. Node

Received 11 December 2015, Revised 2 February 2016, Accepted 10 March 2016.

Correspondence: Christopher A. Barker, MD, Department of Radiation Oncology, Memorial Sloan Kettering Cancer Center, 1275 York Avenue, Box 22, New York, NY 10065, USA. Tel: +1-212-639-8168, Fax: +1-212-717-3104, E-mail: barkerc@mskcc.org *Current affiliation: Department of Radiation Oncology, West Virginia University Medical Center, Morgantown, WV, USA

(c) This is an Open Access article distributed under the terms of the Creative Commons Attribution Non-Commercial License (http://creativecommons.org/ licenses/by-nc/4.0/) which permits unrestricted non-commercial use, distribution, and reproduction in any medium, provided the original work is properly cited.

www.e-roj.org 
basin radiotherapy for other locally advanced and high-risk skin cancers (such as cutaneous squamous cell carcinoma and angiosarcoma) is also likely effective $[3,4]$, but has not been studied in randomized trials.

In the TROG trial noted above, the radiotherapy technique was specified by the investigators [5]. For axilla metastasis, parallel opposed anterior and posterior fields were designed to encompass the axilla and supraclavicular lymph node basins using skeletal anatomy to delineate field borders (Fig. 1A). Similarly for groin metastasis, parallel opposed anterior and posterior fields (with or without a lateral field) were designed to encompass the inguinofemoral and lower external iliac lymph node basins using skeletal anatomy to delineate field borders (Fig. 1B). In the GCCSFD trial noted above, the radiotherapy technique was at the discretion of the radiotherapist [2]. In both of these trials adverse events were not infrequent, which may in part be due to the twodimensional radiotherapy (2D-RT) technique that was utilized.

Since these clinical trials were conducted, there have been significant advances in the planning and delivery of external beam radiotherapy. According to a systematic review, advanced techniques in radiotherapy, such as intensity-modulated radiation therapy (IMRT), may yield fewer clinically significant adverse events and improve disease control, compared with traditional radiotherapy techniques planned in two dimensions (2D-RT) using skeletal anatomy to delineate field borders [6]. Volumetric-modulated arc therapy (VMAT), the next generation of IMRT, may provide further advantages. For those malignancies in which the dosimetric and clinical benefits of IMRT have been clearly demonstrated, IMRT has gained acceptance as a standard part of the clinical treatment of that respective malignancy. While it would be expected that many of the advantages of IMRT and VMAT would hold true in the context of lymph node basin radiotherapy among skin cancer patients, this has not previously been explored in a clinical trial, and as such the magnitude of the relative benefits is unknown. Therefore, we sought to better define the dosimetric benefits of radiotherapy techniques developed over the last 3 decades such as three-dimensional conformal radiation therapy (3D-CRT), IMRT, and VMAT compared to conventional techniques in this clinical context.

\section{Materials and Methods}

\section{Patients and simulation parameters}

With permission from Institutional Review Board, medical records and simulation imaging of 20 patients with regional lymph node metastasis from skin cancer were used for this dosimetric comparison study. Patients were simulated for radiation therapy after therapeutic lymphadenectomy, diagnostic sentinel lymph node biopsy, fine needle aspiration, or excisional biopsy confirming regional metastasis. Radiotherapy simulation was performed with patients lying supine and immobilized in a custom foam form (Soule Medical, Lutz, FL, USA or Smithers Medical Products, North Canton, $\mathrm{OH}, \mathrm{USA}$ ). For simulation of the axilla, the arm was abducted $20^{\circ}-45^{\circ}$, with the hand at or near the lateral iliac crest, in the "akimbo" position, with the neck extended and head rotated away from the target. This position is preferred for patients in this clinical scenario as they typically are elderly and have recently undergone lymphadenectomy thus limiting their ability to abduct the arm. For simulation of the groin, the leg was abducted $15^{\circ}-30^{\circ}$. Computed tomographies (CTs) were acquired with the Brilliance CT Big Bore (Philips Amsterdam, Netherlands) through the region of interest, in 3-mm slices. Iodinated intravenous contrast $\left(100-150 \mathrm{~cm}^{3}\right.$ at $1-2 \mathrm{~cm}^{3} / \mathrm{s}$; Omnipaque, GE Healthcare, Princeton, NJ, USA) was administered via the antecubital vein contralateral to the

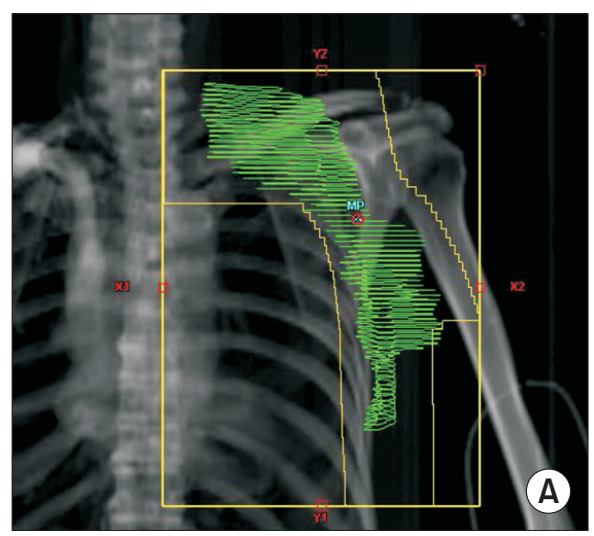

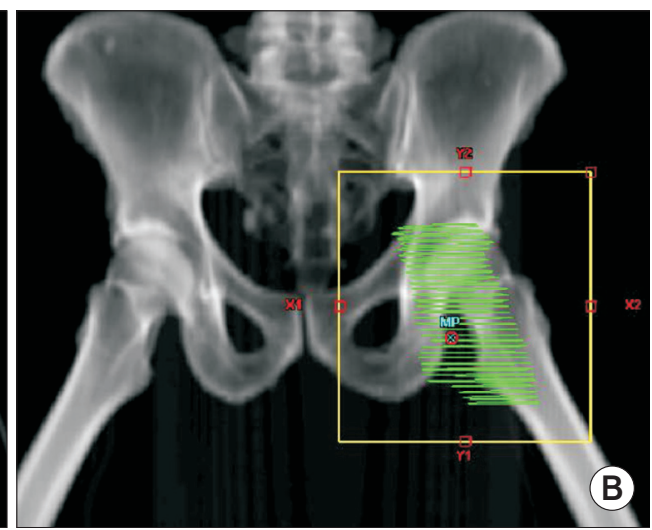

B
Fig. 1. Digitally reconstructed radiographs with representative twodimensional radiotherapy fields (with clinical target volume superimposed in green) for (A) axilla and (B) groin radiotherapy for high-risk or locally advanced skin cancer. 
target in patients without medical contraindications.

\section{Clinical target volume delineation and planning target volume generation}

The clinical target volumes (CTVs) were delineated and agreed upon by two radiation oncologists. Each case included only nodal regions, and did not encompass the primary tumor site. The CTVs for the axilla and groin regions were delineated to correspond with the groin and axilla node basins targeted in the TROG 96.06 and 02.01 trials [1,5], and the Radiation Therapy Oncology Group (RTOG) consensus guidelines (for breast [7] and anorectal [8] cancer). In all cases, a planning target volume (PTV) expansion of $1 \mathrm{~cm}$ was applied isotropically around the CTV.

\section{Organ at risk delineation}

Organs at risk (OARs) were delineated and agreed upon by two radiation oncologists. OARs in the axilla region were contoured per the RTOG atlas for thoracic radiotherapy [9]. OARs for the groin region were contoured per the RTOG consensus contouring guidelines for normal tissues of the pelvis [10]. The ipsilateral brachial plexus, lumbosacral plexus, and larynx were delineated according to published guidelines [11-13]. The pelvic bones included the sacrum, coccyx and bilateral ilium, ischium, and pubis bones. The skin was defined as the outermost $5 \mathrm{~mm}$ of the body.

\section{Radiation dose prescription}

Because the radiation dose prescription for high-risk and locally advanced skin cancer varies based on disease and treatment intent, and performing radiotherapy plans for all of these scenarios was not feasible, a moderate dose of 56 Gy in 28 fractions was chosen for planning purposes. Each PTV was planned to receive this prescription dose.

\section{Treatment planning}

All treatment plans were generated by a medical physicist exclusively for the purpose of this study. All optimizations and dose calculations were performed in the Eclipse (Varian Medical Systems, Palo Alto, CA, USA) treatment-planning system using an Anisotropic Analytical Algorithm (ver. 11).

The 2D-RT plans were generated based on bone anatomy in accordance with TROG 96.01 specifications [5]. Both the axilla and groin plans utilized parallel opposed anterior-posterior fields of mixed-energy photons (15 MV for posteroanterior field, 6 MV for anteroposterior field) weighted 50:50 without any compensators or wedges. The dose was prescribed to midplane depth at the center of the field.

3D-CRT plans were generated using 5-6 mixed-energy (6 and $15 \mathrm{MV}$ ) photon fields, at least $30^{\circ}$ apart. Axilla plans used three anterior oblique fields and 3 posterior oblique fields to avoid the ipsilateral arm. Groin plans used 3 anterior oblique fields, 1 lateral field, and 1 posterior oblique field. Multileaf collimation was set using a $0.8-\mathrm{cm}$ margin expansion of the beams eye view projection of the PTV. Collimator angles and beam weights were manually adjusted to optimize the dose distribution and meet OAR constraints. Wedges were used for the groin plans but were not used for the axilla plans due to field size restrictions of physical and dynamic wedges.

IMRT plans were inverse planned using 5-7 mixedenergy ( 6 and $15 \mathrm{MV}$ ) photon fields arranged similarly to the corresponding 3D-CRT plans. VMAT plans were generated using $6 \mathrm{MV}$ photons, with arcs spanning an angle range $\left(200^{\circ}-210^{\circ}\right.$ for axilla, $150^{\circ}-160^{\circ}$ for groin) similar to the corresponding 3D-CRT and IMRT plans. A collimator angle of $10^{\circ}$ or $350^{\circ}$ was used to minimize tongue and groove effects. Groin plans used two arcs without an avoidance sector whereas axilla plans utilized a total of four arcs (2 clockwise and 2 counterclockwise with a 2 -cm overlapping region between the jaw opening of the arcs). The latter approach was used for the axilla because the PTV was larger than $25 \mathrm{~cm}$ and most linear accelerators cannot accommodate dynamically collimated arc fields larger than $15 \mathrm{~cm}$. For the axilla, each arc had an avoidance sector angle range of $80^{\circ}-90^{\circ}$ to avoid the ipsilateral arm on the side of body.

3D-CRT, IMRT, and VMAT plans were normalized such that 95\% of the PTV received 100\% of the prescription dose. Dose distributions were designed with the intention of respecting the OAR dose-volume constraints shown in Table 1, and were selected for inclusion in this study based on their use in active or recently published RTOG protocols. In cases where more than one constraint has been commonly utilized in RTOG protocols (e.g., esophagus $D_{\text {meanı }}$ lung $D_{20}$ and lumbosacral plexus $\left.D_{\max }\right)$, only the more stringent constraint was used in optimization. In cases where the RTOG constraint for a given organ exceeded the prescription dose in this study (e.g., brachial plexus), an effort was made to avoid hot spots in that organ. Of note, although the skin was included in the subsequent analysis, no specific skin constraints were used for treatment planning purposes.

\section{Dosimetric analysis}

For the purposes of dosimetric analysis, $V x$ was defined as the volume of a structure receiving more than dose $x$ (Gy). Dy was 
Table 1. Organ at risk dose-volume constraints utilized in this study, along with the protocols from which they are derived

\begin{tabular}{|c|c|c|c|}
\hline Organ & Volume & Dose (Gy) & Protocol \\
\hline \multirow[t]{2}{*}{ Brachial plexus } & $\mathrm{D}_{\max }$ & 66 & RTOG 0619, 0617, 0615 \\
\hline & $\mathrm{D}_{5 \%}$ & 60 & RTOG 0619 \\
\hline \multirow[t]{2}{*}{ Esophagus } & $D_{\text {mean }}$ & 30 & RTOG 1016 \\
\hline & $\mathrm{D}_{\text {mean }}$ & 34 & RTOG 0623, 0617 \\
\hline \multirow[t]{3}{*}{ Heart } & $\mathrm{D}_{33}$ & 50 & RTOG 0436 \\
\hline & $D_{67}$ & 45 & RTOG 0435, 0623, 0617 \\
\hline & $D_{100}$ & 40 & RTOG 0435, 0623, 0617 \\
\hline Proximal humerus & $D_{50}$ & 50 & RTOG 0630 \\
\hline \multirow[t]{2}{*}{ Larynx } & $D_{\max }$ & 45 & RTOG 0619, 0615, 0522 \\
\hline & $D_{\text {mean }}$ & 20 & RTOG 1016 \\
\hline Liver & $\mathrm{D}_{50}$ & 35 & RTOG 0436 \\
\hline \multirow[t]{3}{*}{ Total lung } & $V_{37}$ & $20 \%$ & RTOG 0617, 0623 \\
\hline & $\mathrm{V}_{20}$ & $20 \%$ & RTOG 0630 \\
\hline & $D_{\text {mean }}$ & 20 & RTOG 0617 \\
\hline Spinal canal & $D_{\max }$ & 45 & RTOG 0623, 0615 \\
\hline \multirow[t]{2}{*}{ Bladder } & $D_{50}$ & 35 & RTOG 0529 \\
\hline & $\mathrm{D}_{5}$ & 50 & RTOG 0529 \\
\hline \multirow[t]{2}{*}{ Bowel bag } & $D_{20 c c}$ & 45 & RTOG 0529 \\
\hline & $D_{200 c c}$ & 30 & RTOG 0529 \\
\hline \multirow[t]{2}{*}{ Genitalia } & $D_{50}$ & 20 & RTOG 0529 \\
\hline & $D_{5}$ & 40 & RTOG 0529 \\
\hline \multirow[t]{3}{*}{ Lumbosacral plexus } & $\mathrm{D}_{\max }$ & 66 & $\begin{array}{l}\text { Extrapolation from } \\
\text { RTOG 0619, 0617, } 0615\end{array}$ \\
\hline & $D_{\max }$ & 60 & RTOG 0522 \\
\hline & $D_{5 \%}$ & 60 & RTOG 0619 \\
\hline \multirow[t]{2}{*}{ Proximal femur (weight bearing bone) } & $D_{50}$ & 50 & RTOG 0630 \\
\hline & $D_{5}$ & 60 & RTOG 0630 \\
\hline Pelvic bones & $D_{37}$ & 40 & RTOG 0418 \\
\hline Anorectum & $D_{50}$ & 30 & RTOG 0630 \\
\hline
\end{tabular}

RTOG, Radiation Therapy Oncology Group.

defined as the minimum dose to y percent (or $\mathrm{cm}^{3}$ as indicated) of structure. $D_{\max }$ was defined according to the aforementioned RTOG protocols in Table 1 as the highest dose within a volume of $0.03 \mathrm{~cm}^{3}$ of a given structure. Dosimetric parameters used to compare PTV coverage included the homogeneity index, conformity index, and gradient index. The homogeneity index was calculated as $\left(D_{2}-D_{98}\right) /($ prescription dose in cGy) $\times 100$ with a value closer to zero indicating greater homogeneity. The conformity index was calculated as the ratio of the volume within the prescription isodose surface to the PTV, with a value closer to unity indicating greater conformity. The gradient index was calculated as the ratio of the 50\% isodose volume to the PTV, with a smaller gradient index indicating higher dose gradients around the target. Dosimetric parameters to evaluate OAR doses are presented in Table 1. The normal tissue integral dose was also calculated for all plans as the product of the mean dose (Gy) to a region encompassing the normal tissue (excluding the PTV) inside the scanned region, and the volume (L) of that region. Finally, because the most common high grade adverse event after lymph node basin RT is skin injury, the skin $D_{\text {max }} D_{50 c c 1}$ and $D_{300 c c}$ were evaluated.

\section{Statistics}

For both the axilla and groin plans, the mean and median value of each of the above dose-volume histogram parameters for both techniques are presented. Two-tailed Student t-tests were used to compare the individual techniques. Statistical analysis was performed using SPSS ver. 20.0 (IBM, Armonk, NY, USA). A p-value of less than 0.05 was considered statistically significant.

\section{Results}

The simulation images of 20 patients (10 axilla, 10 groin) were 

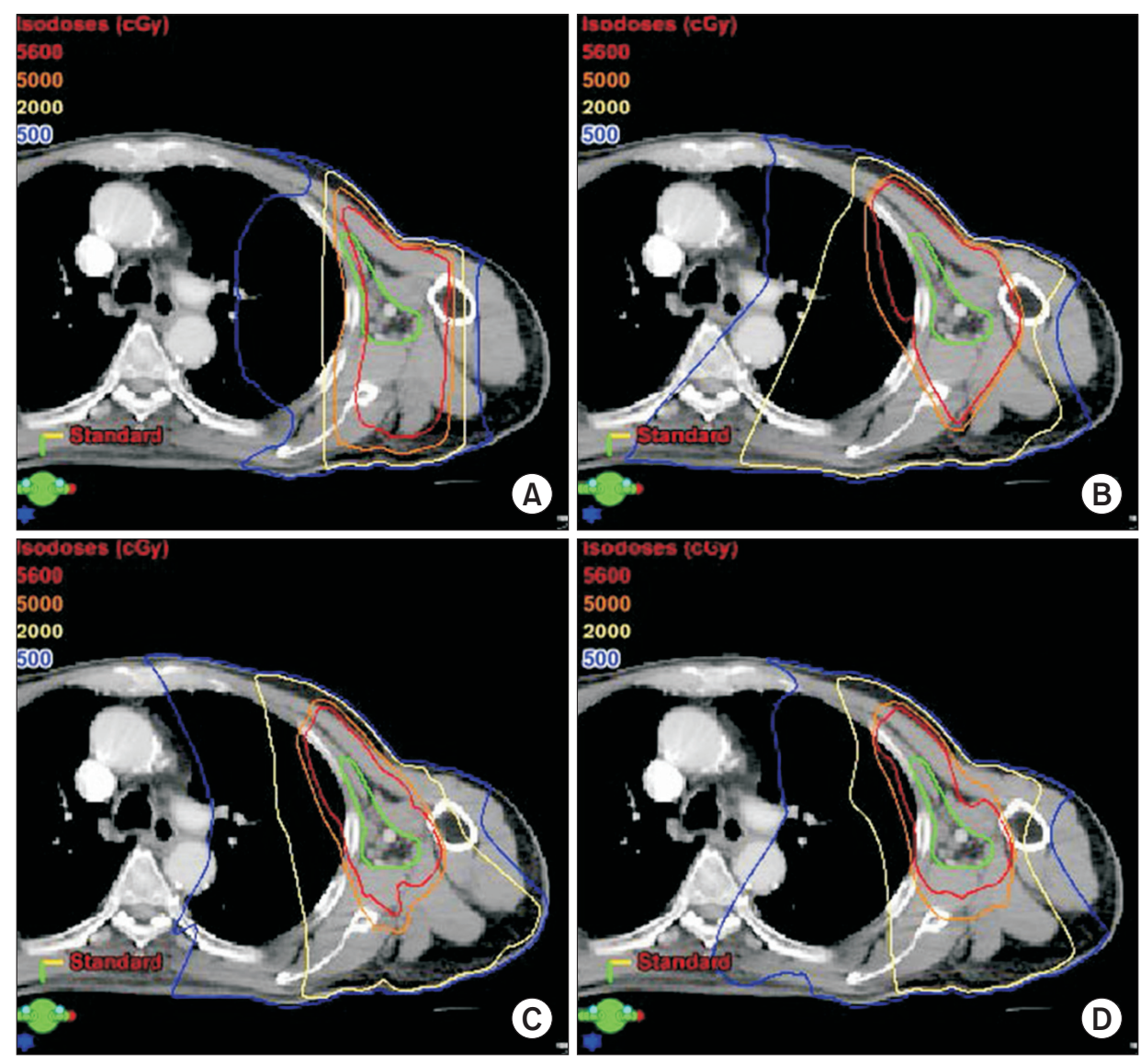

Fig. 2. Computed tomography scans with representative isodose distributions for (A) two-dimensional radiotherapy, (B) three-dimensional conformal radiotherapy, (C) intensity-modulated radiotherapy, and (D) volumetric-modulated arc radiotherapy for high-risk and locally advanced skin cancer involving the axilla. Clinical target volume indicated in green.

used to generate the treatment plans for this analysis. The mean PTV volume of the axilla was 1,215.1 $\mathrm{cm}^{3}$ (median, 1,261.0 $\mathrm{cm}^{3}$ ) and the mean PTV volume of the groin was $422.8 \mathrm{~cm}^{3}$ (median, $412.5 \mathrm{~cm}^{3}$ ). The median age of the patients studied was 72 years (range, 40 to 90 years). More of the patients were men (70\%). Tumor histologies included Merkel cell carcinoma (65\%), melanoma (25\%), squamous cell carcinoma (5\%), and basal cell carcinoma (5\%). Lymph node metastasis was confirmed by therapeutic lymphadenectomy (40\%), sentinel lymph node biopsy (35\%), excisional biopsy (15\%), and fine needle aspiration (10\%).

Dosimetric analyses of the 4 treatment-planning techniques for the axilla are shown in Fig. 2 and Table 2. The majority of OAR constraints were met for all techniques, with the exceptions that the average 2D-RT and 3D-CRT plans did not achieve the brachial plexus $D_{5}$ constraint and 3D-CRT plans did not achieve the lung $\mathrm{V}_{20}$ constraint. Compared to 2D-RT plans, 3D-CRT plans were associated with statistically significant improvements in the homogeneity index and skin $D_{50 c c}$ and $D_{300 c c}$ at the expense of significantly higher lung doses using 3D-CRT. IMRT and VMAT resulted in statistically significant improvements compared to $2 \mathrm{D}-\mathrm{RT}$ and $3 \mathrm{D}-\mathrm{CRT}$ plans for the majority of parameters. Of note, the lung $V_{5}$ was higher using IMRT or VMAT compared to 2D-RT, but the lung $V_{20}$ and $D_{\text {mean }}$ were statistically no different to that of $2 \mathrm{D}-\mathrm{RT}$, and the lung $V_{37}$ was improved using IMRT or VMAT. The humerus $D_{\text {mean }}$ and spinal canal $D_{\max }$ were also greatly improved with IMRT or VMAT. Differences that were smaller magnitude but statistically significant were observed between VMAT and IMRT as well, as per Table 2.

Dosimetric analyses of the 4 treatment-planning techniques for the groin are shown in Fig. 3 and Table 3. The majority of OAR constraints were met for all techniques, with the exceptions that the average 2D-RT plan did not achieve the bowel bag $D_{20 c c}$ or femur $D_{50}$ constraints, and the average 3D-CRT plan did not achieve the bowel bag $D_{20 c c}$. Regarding PTV coverage, an inverse relationship between 2D-RT and 3D-CRT plans was observed for the groin as compared to the axilla, with 3D-CRT plans having superior conformity index and gradient index than 2D-RT plans in the groin without any difference in homogeneity index. Large magnitude statistically significant improvements in OAR sparing using 3D-CRT compared to 2D-RT were observed for the anorectum $D_{\max }$ bowel bag $D_{200 c c}$ femur $D_{50}$, genitalia $D_{\max }$ skin $D_{50 c c 1}$ 


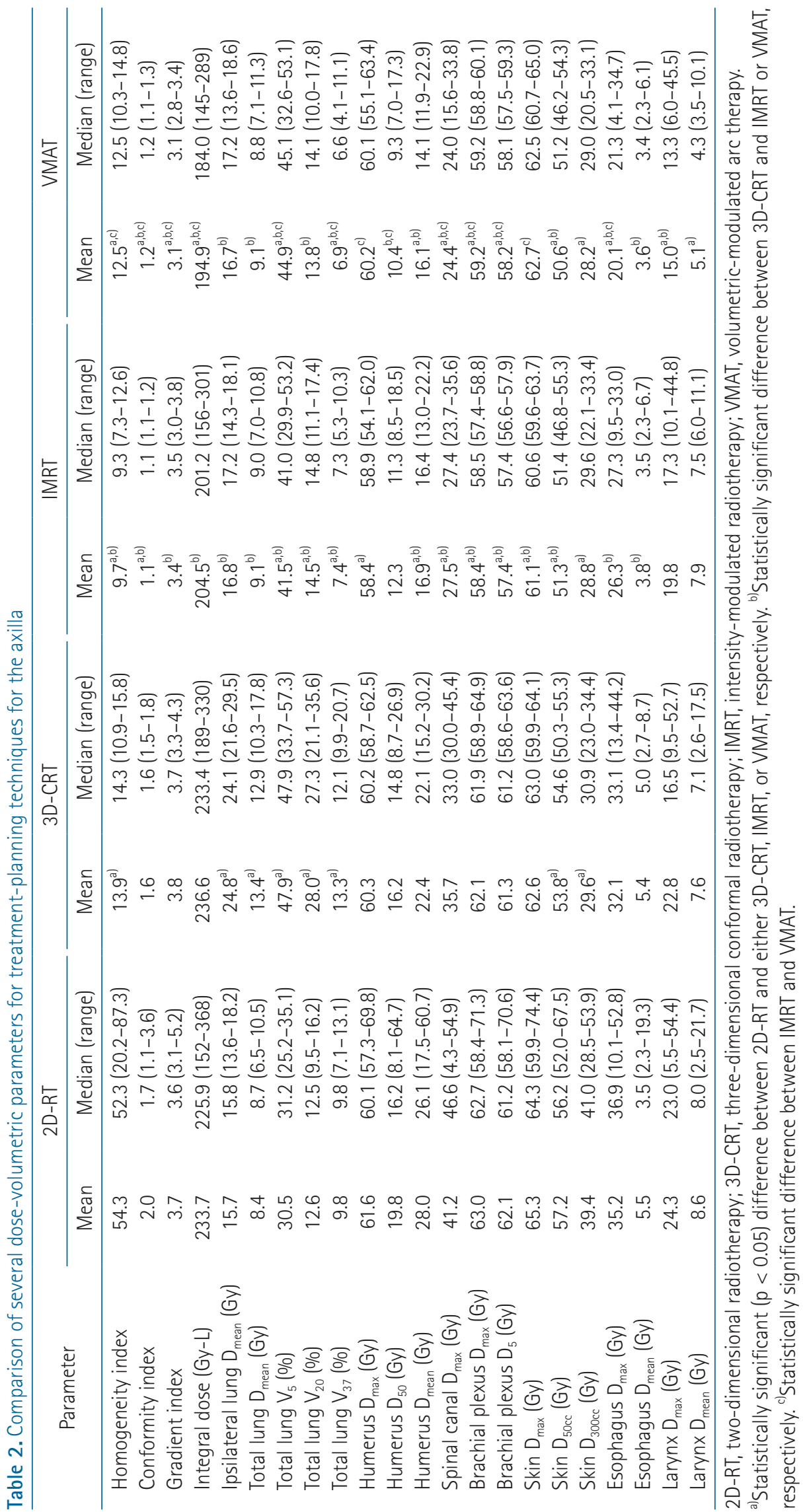



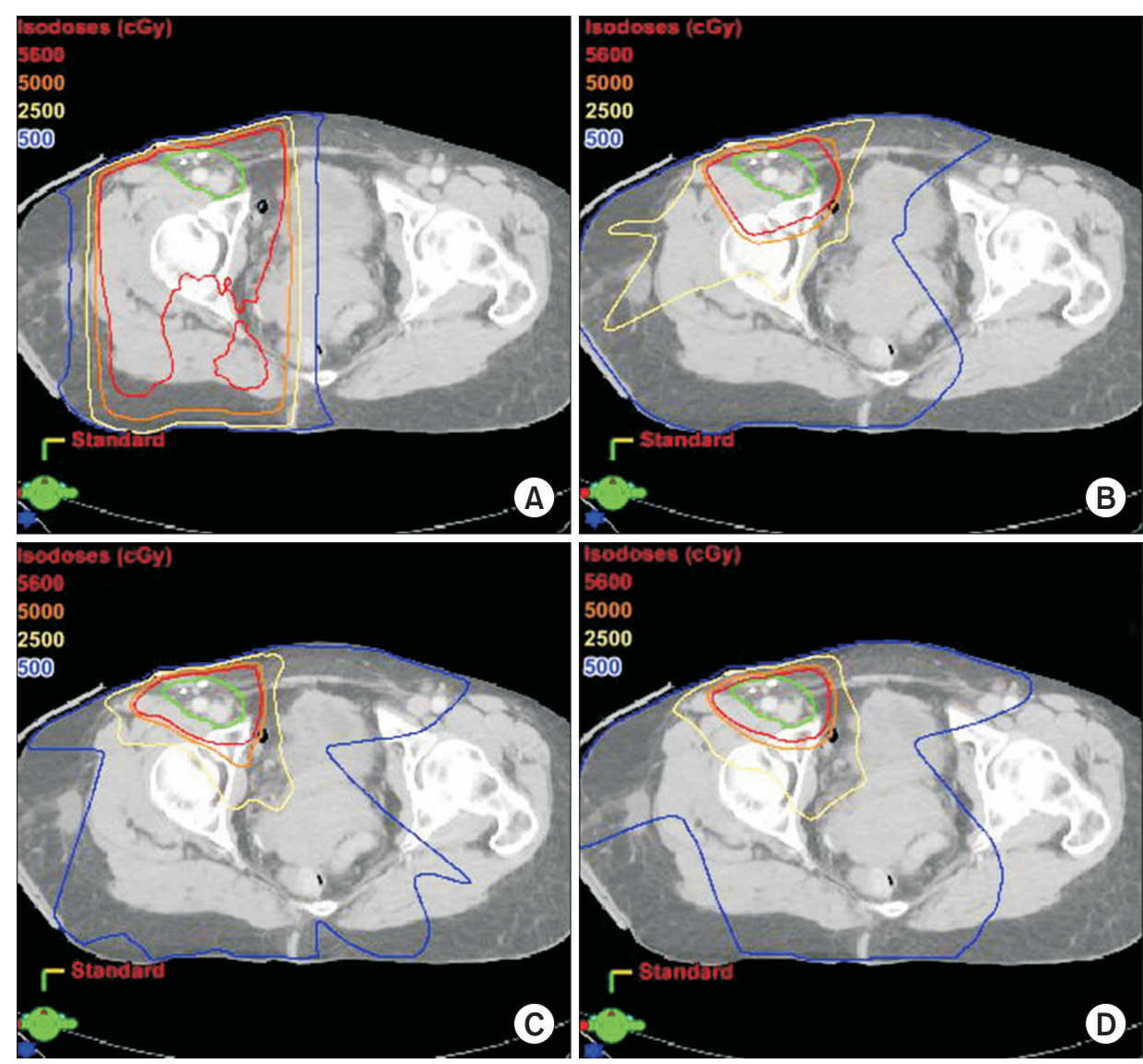

Fig. 3. Computed tomography scans with representative isodose distributions for (A) two-dimensional radiotherapy, (B) three-dimensional conformal radiotherapy, (C) intensity-modulated radiotherapy, and (D) volumetric-modulated arc radiotherapy for high-risk and locally advanced skin cancer involving the groin. Clinical target volume indicated in green.

and lumbosacral plexus $D_{\max }$. The use of IMRT and VMAT led to further dosimetric improvements in most endpoints. Anorectum doses were somewhat higher with IMRT and VMAT compared to $3 \mathrm{D}-\mathrm{CRT}$, though within constraints. As for the axilla plans, the magnitude of differences between IMRT and VMAT was again small, as per Table 3.

\section{Discussion and Conclusion}

IMRT is an inverse treatment planning process that optimizes the intensity distribution of a set of beams according to dosevolume histogram objectives chosen by planners, allowing for highly conformal treatment of a target while sparing OARs. As such, IMRT is particularly valuable when a target volume has significant convexity or concavity, or is in close proximity or overlapping an OAR. VMAT allows for variation in the dose rate, speed of gantry rotation, and multi-leaf positions during rotation of the gantry in a full $360^{\circ}$ arc, possibly contributing to further dosimetric advantages as compared to fixedbeam IMRT for some disease sites. This study was designed to compare four radiotherapy techniques in the treatment of the axilla and groin lymph node basins for locally advanced or high-risk skin cancer. While some minor differences between the IMRT and VMAT techniques were observed, we have shown for the first time in this clinical context that either IMRT or VMAT is superior to 2D-RT and 3D-CRT plans for the majority of the clinically significant dosimetric parameters evaluated. Clinical outcome studies to identify whether these dosimetric improvements translate into fewer side effects and increased local control are warranted.

The observed advantages and disadvantages of each treatment planning technique are not only dependent on the technique itself but also the properties of the target volume and site of treatment, thus leading to some discrepancies in the observed differences between planning techniques for the axilla and groin. For instance, the greatly inferior homogeneity of the 2D-RT plans for the axilla is most likely due to the fact that the axilla target volume was larger, longer, and had greater curvature than the groin target volume, and also the greater variation in the thickness of tissue surrounding the axilla across the craniocaudal length of the target. 3D-CRT, IMRT, and VMAT are better able to account for these differences in thickness and provide a more homogenous dose. However in doing so, more fields must inherently cross the lung, thus 


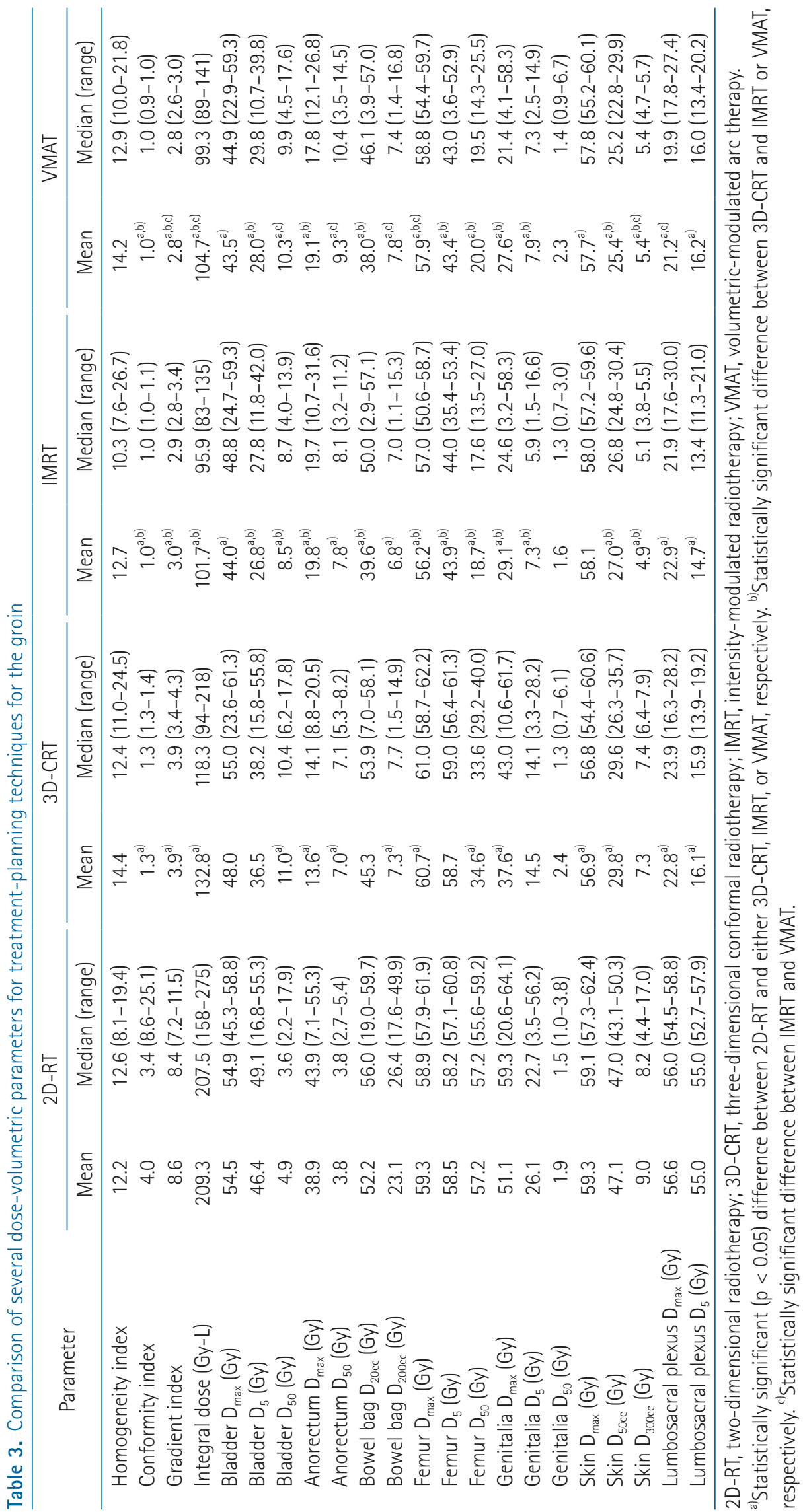


leading to the elevations in the low-dose region of the lung dose volume histogram (DVH) (as represented by higher $\mathrm{V}_{5}$ ). By modulating the dose, IMRT and VMAT were able to achieve more acceptable lung $V_{20}$ and $V_{37}$, which were comparable to the $2 \mathrm{D}-\mathrm{RT}$ plans. The smaller groin target volume on the other hand did not present the same issues of homogeneous dose coverage using AP/PA fields, though the greater conformity and dose fall-off with the more advanced techniques again led to significant benefits in OAR sparing. Our findings are consistent with previous clinical studies that have also found that more advanced radiotherapy techniques are associated with lower doses to OARs, and a decreased frequency and severity of adverse events. The earliest examples of this came with the transition from 2D-RT to 3D-CRT for prostate cancer. In randomized trials, rates of acute and late radiation proctitis were reduced with 3D-CRT $[14,15]$. Investigators later reported that IMRT offered advantages over 3D-CRT, with greater sparing of the bowel, rectum, and bladder in patients treated with pelvic radiotherapy for prostate cancer, with fewer adverse events [16]. In breast cancer, randomized trials demonstrated less acute dermatitis and late skin induration when comparing 2D-RT to IMRT $[17,18]$. Likewise, in head and neck cancers, several randomized trials have demonstrated that IMRT can spare parotid gland injury better than 2D-RT [19-21]. These studies have all demonstrated the clinical value of advanced techniques.

As demonstrated in the TROG clinical trials, Iymphedema and skin or subcutaneous toxicity are not infrequent using 2DRT. In the phase II study (TROG 96.06) with long-term followup, grade 3 or 4 lymphedema was noted in 9 of 109 patients (8.3\%) treated with axilla radiotherapy, and 19 of 48 patients $(39.6 \%)$ treated with groin radiotherapy. Long-term results of the randomized trial suggested grade 2 or higher subcutaneous tissue fibrosis was common (approximately 60\%) [22]. The dosimetric analyses presented here indicate that significantly improved conformity and gradient indices and lower skin dose associated with IMRT and VMAT may yield fewer skin and subcutaneous adverse events by irradiating a smaller volume of nontarget OARs. Normal tissue complications probability models can also be used to help estimate the expected clinical outcomes from the observed dosimetric benefits. For instance, the 2D-RT and 3D-CRT mean brachial plexus D5 exceeded the $60 \mathrm{~Gy}$ constraint and would be expected to be associated with a $5 \%$ risk of brachial plexopathy within 5 years [23]. The higher mean lung dose for the 3D-CRT plans would also be associated with an approximately 5\% increased risk of symptomatic pneumonitis compared to the other techniques
[24]. The elevated femur $D_{50}$ for 2D-RT plans would carry a $5 \%$ risk of necrosis or fracture within the next 5 years [23]. Finally, the 2D-RT and 3D-CRT plans exceeding the bowel constraints would likely result in a similar 15\% increase in late grade 3 or higher gastrointestinal toxicity as observed in RTOG 0529 for anal cancer [25].

Further improvement in regional disease control may also be possible with the use of advanced treatment techniques. In the GCCSFD study of Merkel cell carcinoma, regional recurrence was not observed after radiotherapy. This is probably related to the relative radiosensitivity of Merkel cell carcinoma compared with other skin cancers. However, radiotherapy technique was left at the discretion of the investigators and may have involved advanced techniques. In the TROG studies, 2D-RT was associated with a cumulative incidence of lymph node relapse of 19\%. In our analysis, based on the target delineation using $\mathrm{CT}$, we found that areas of the axilla PTV may have been underdosed using 2D-RT, whereas IMRT and VMAT were associated with more homogeneous PTV coverage. Whether this would result in improved regional disease control remains to be determined, but support of this hypothesis is derived from a recent retrospective analysis that suggested a lymph node relapse rate lower than what was observed in the TROG trials with limited side effects when using adjuvant IMRT for cutaneous melanoma of the head and neck [26].

The relative benefits of the more advanced IMRT and VMAT modalities must also be weighed against their costs. Accounting for the increased complexity of simulation, treatment planning, image guidance, and quality assurance with IMRT and VMAT plans, the estimated overall cost for a 28 fraction course of each technique in the United States are \$8,363 for 2D-RT, \$14,693 for 3D-CRT, and \$21,994 for IMRT/ VMAT. Whether the extra costs associated with IMRT and VMAT are justified by their dosimetric benefits is uncertain, and should be assessed on an individual basis depending on the extent of the patient's malignancy, severity of comorbidities, and overall life expectancy. In situations in which IMRT and VMAT are not available, it appears from our data that 3D-CRT would be preferred to 2D-RT for the groin based on the large magnitude improvements in the high-dose region portion of the DVH for most organs, but for the axilla this does not necessarily appear to be the case and 2D-RT may be preferred. Since IMRT and VMAT have a similar cost and the dosimetric differences between them were less prominent in this study, we suggest that institutional expertise in generating a high quality plan should take priority in deciding which one to use for most patients. 
There are several limitations to this study. First, as a treatment-planning study in which none of the plans generated were used to treat patients, we cannot assess the impact of our dosimetric findings on clinical outcomes. However, correlation between OAR dosimetry and toxicity has been well studied, and we relied on published constraints from cooperative group protocols to guide plan generation and assessment [27]. Second, we chose to study only axilla and groin radiotherapy, because of the extensive literature that has demonstrated the advantages of IMRT in head and neck cancer [19-21]. Third, any treatment planning study is inherently dependent on the planner and their choice of beam angles, energy, and number of fields. For instance, some planners may in their practice use only 3-4 fields for a 3D-CRT plan in the axilla rather than the 6 fields used in this study, and the angles and weighting of these fields would certainly have implications on lung dose and PTV homogeneity/conformity. We elected to use identical numbers and angles of fields for IMRT and 3D-CRT plans (5 fields in the groin and 6 fields in the axilla) in this study in order to ensure as fair a comparison as possible, and try to isolate the effect of modulation while keeping other factors constant. If anything, using more fields in a 3D-CRT plan than may be typical should allow for greater flexibility in PTV coverage and OAR sparing than would be achievable with fewer fields. Finally, radiation dose and fractionation for locally advanced and high-risk skin cancer spans a wide range (46-70 Gy in 2 Gy fractions) depending on the specific disease and treatment context. The higher the prescription dose, the more challenging it would be to generate a plan that meets the goals and constraints for the PTV and OARs, respectively, regardless of the treatment technique used. We addressed this issue by choosing an intermediate dose (56 Gy) in this study, which is an equivalent dose in $2 \mathrm{~Gy}$ fractions to what was used in the TROG trials (using linear-quadratic modeling and an alpha/beta of 0.6 for melanoma). Our findings should be scalable to regimens using a higher or lower prescription dose.

In conclusion, this study was designed to compare four radiotherapy techniques for locally advanced and high-risk skin cancer. We found that IMRT and VMAT are better able to deliver the radiation dose to the target than $2 \mathrm{D}-\mathrm{RT}$ or $3 \mathrm{D}-\mathrm{CRT}$, with lower doses to nearby OARs of radiation injury. These results suggest that fewer side effects from radiotherapy and improved regional disease control may be possible using more advanced techniques such as IMRT and VMAT. These data provide support for clinical trials of advanced radiotherapy techniques for locally advanced skin cancers.

\section{Conflict of Interest}

No potential conflict of interest relevant to this article was reported.

\section{Acknowledgments}

This study was funded in part through the National Institutes of Health/National Cancer Institute Cancer Center Support Grant (P30 CA008748).

\section{References}

1. Burmeister BH, Henderson MA, Ainslie J, et al. Adjuvant radiotherapy versus observation alone for patients at risk of lymph-node field relapse after therapeutic lymphadenectomy for melanoma: a randomised trial. Lancet Oncol 2012;13:58997.

2. Jouary T, Leyral C, Dreno B, et al. Adjuvant prophylactic regional radiotherapy versus observation in stage I Merkel cell carcinoma: a multicentric prospective randomized study. Ann Oncol 2012;23:1074-80.

3. O'Hara J, Ferlito A, Takes RP, et al. Cutaneous squamous cell carcinoma of the head and neck metastasizing to the parotid gland: a review of current recommendations. Head Neck 2011;33:1789-95.

4. Mendenhall WM, Mendenhall CM, Werning JW, Reith JD, Mendenhall NP. Cutaneous angiosarcoma. Am J Clin Oncol 2006;29:524-8.

5. Burmeister BH, Mark Smithers B, Burmeister E, et al. A prospective phase II study of adjuvant postoperative radiation therapy following nodal surgery in malignant melanomaTrans Tasman Radiation Oncology Group (TROG) Study 96.06. Radiother Oncol 2006;81:136-42.

6. Veldeman L, Madani I, Hulstaert F, De Meerleer G, Mareel M, De Neve W. Evidence behind use of intensity-modulated radiotherapy: a systematic review of comparative clinical studies. Lancet Oncol 2008:9:367-75.

7. Li XA, Tai A, Arthur DW, et al. Variability of target and normal structure delineation for breast cancer radiotherapy: an RTOG Multi-Institutional and Multiobserver Study. Int J Radiat Oncol Biol Phys 2009;73:944-51.

8. Myerson RJ, Garofalo MC, El Naqa I, et al. Elective clinical target volumes for conformal therapy in anorectal cancer: a RTOG consensus panel contouring atlas. Int J Radiat Oncol Biol Phys 2009;74:824-30.

9. Kong FM, Ritter T, Quint DJ, et al. Consideration of dose limits for organs at risk of thoracic radiotherapy: atlas for lung, proximal bronchial tree, esophagus, spinal cord, ribs, and 
brachial plexus. Int J Radiat Oncol Biol Phys 2011;81:1442-57.

10. Gay HA, Barthold HJ, O'Meara E, et al. Pelvic normal tissue contouring guidelines for radiation therapy: a Radiation Therapy Oncology Group consensus panel atlas. Int J Radiat Oncol Biol Phys 2012;83:e353-62.

11. Hall WH, Guiou M, Lee NY, et al. Development and validation of a standardized method for contouring the brachial plexus: preliminary dosimetric analysis among patients treated with IMRT for head-and-neck cancer. Int J Radiat Oncol Biol Phys 2008;72:1362-7.

12. Galloway TJ, Amdur RJ, Liu C, Yeung AR, Mendenhall WM. Revisiting unnecessary larynx irradiation with whole-neck IMRT. Pract Radiat Oncol 2011;1:27-32.

13. Yi SK, Mak W, Yang CC, et al. Development of a standardized method for contouring the lumbosacral plexus: a preliminary dosimetric analysis of this organ at risk among 15 patients treated with intensity-modulated radiotherapy for lower gastrointestinal cancers and the incidence of radiationinduced lumbosacral plexopathy. Int J Radiat Oncol Biol Phys 2012;84:376-82.

14. Koper PC, Stroom JC, van Putten WL, et al. Acute morbidity reduction using 3D-CRT for prostate carcinoma: a randomized study. Int J Radiat Oncol Biol Phys 1999;43:727-34.

15. Dearnaley DP, Khoo VS, Norman AR, et al. Comparison of radiation side-effects of conformal and conventional radiotherapy in prostate cancer: a randomised trial. Lancet 1999;353:267-72.

16. Ashman JB, Zelefsky MJ, Hunt MS, Leibel SA, Fuks Z. Whole pelvic radiotherapy for prostate cancer using 3D conformal and intensity-modulated radiotherapy. Int J Radiat Oncol Biol Phys 2005;63:765-71.

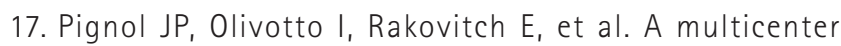
randomized trial of breast intensity-modulated radiation therapy to reduce acute radiation dermatitis. J Clin Oncol 2008;26:2085-92.

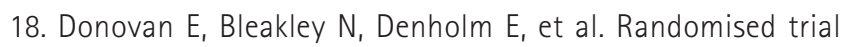
of standard 2D radiotherapy (RT) versus intensity modulated radiotherapy (IMRT) in patients prescribed breast radiotherapy. Radiother Oncol 2007;82:254-64.
19. Kam MK, Leung SF, Zee $B$, et al. Prospective randomized study of intensity-modulated radiotherapy on salivary gland function in early-stage nasopharyngeal carcinoma patients. J Clin Oncol 2007;25:4873-9.

20. Pow EH, Kwong DL, McMillan AS, et al. Xerostomia and quality of life after intensity-modulated radiotherapy vs. conventional radiotherapy for early-stage nasopharyngeal carcinoma: initial report on a randomized controlled clinical trial. Int J Radiat Oncol Biol Phys 2006;66:981-91.

21. Nutting $\mathrm{CM}$, Morden JP, Harrington KJ, et al. Parotid-sparing intensity modulated versus conventional radiotherapy in head and neck cancer (PARSPORT): a phase 3 multicentre randomised controlled trial. Lancet Oncol 2011;12:127-36.

22. Henderson MA, Burmeister BH, Ainslie J, et al. Adjuvant lymph-node field radiotherapy versus observation only in patients with melanoma at high risk of further lymph-node field relapse after lymphadenectomy (ANZMTG 01.02/TROG 02.01): 6-year follow-up of a phase 3, randomised controlled trial. Lancet Oncol 2015;16:1049-60.

23. Emami B, Lyman J, Brown $A$, et al. Tolerance of normal tissue to therapeutic irradiation. Int J Radiat Oncol Biol Phys 1991 21:109-22.

24. Marks LB, Yorke ED, Jackson A, et al. Use of normal tissue complication probability models in the clinic. Int J Radiat Oncol Biol Phys 2010;76(3 Suppl):S10-9.

25. Kachnic LA, Winter K, Myerson RJ, et al. RTOG 0529: a phase 2 evaluation of dose-painted intensity modulated radiation therapy in combination with 5-fluorouracil and mitomycin-C for the reduction of acute morbidity in carcinoma of the ana canal. Int J Radiat Oncol Biol Phys 2013;86:27-33.

26. Hallemeier $C L$, Garces $Y$ I, Neben-Wittich MA, et al. Adjuvant hypofractionated intensity modulated radiation therapy after resection of regional lymph node metastases in patients with cutaneous malignant melanoma of the head and neck. Pract Radiat Oncol 2013;3:e71-7.

27. Radiation Therapy Oncology Group. RTOG protocol table [Internet]. Philadelphia, PA: Radiation Therapy Oncology Group; c2016 [cited 2016 Mar 18]. Available from: http://www.rtog. org/ClinicalTrials/ProtocolTable.aspx. 